\title{
Research and Application of Online monitoring system in sewage treatment Based on Web Service
}

\author{
Chenglian Wu \& Hong Liang \& Yin Qian \& Yuan Zhang \\ School of Information Engineering, Yunnan University, Kunming, China
}

Keywords: Sewage Treatment; Online monitoring; Web Service; ArcGIS Server JavaScript API.

\begin{abstract}
The primary aim of online monitoring system of sewage treatment in Kunming, this system is built basing on Web Service Structures, is real-time monitoring of water quality parameters so that the changing of water quality can be easily known. The mission of this system focuses on receiving and managing the real-time data from each monitoring points, and integrates the management function of GIS by combining with ArcGIS Server JavaScript API. The integrated management of the water quality monitor and data management analysis making it possible that the constantly and accurately date can be provided for the department of sewage treatment plant. At the same time, the objective of improving work efficiency and effectively control of the water quality can be achieved.
\end{abstract}

\section{Introduction}

As one of the most important city infrastructures, the development level of urban sewage treatment system directly affects the urban dweller's health and the quality of their life. Because of sewage treatment industry develops lately, the practical problems, such as the backward technology, the lower processing rate, are still exists. The traditional mode of sewage treatment is the workers extract the water quality monitoring data regular or irregular at monitoring base station, then, a series of data summarization, arrangement and comprehensive analysis will be done. The manual process is the characteristics of lower reliability and long period. So much complicated the data is, the manual-work cannot calculate and analyze the data incorrectly. Therefore, a monitoring system on sewage treatment is necessary for us to make automatically uploaded the monitoring data and statistical analysis the data possible. The sewerage treatment system that base of Web Service is the automatic, informatization and scientific management system. It's not only change the mode of water quality monitoring in Kunming, but also provide the exactly and constantly data for user.

\section{System design}

\subsection{System structure}

The system structure consists of data collection, transmission, application service and customer service. As shown in the figure 1.

The function of collection layer is responsible for the data collection, which include water COD, ANDAN, $\mathrm{PH}$ and rate of flow.

The transmission layer is mainly responsible for transfer monitoring data information by computer network, backbone network and the two level communication networks.

The function of application layer is managing the information of monitoring data and sewage treatment plants.

The customer service layer is the interface that the users use. The function of this layer is calling the function of online monitoring system and sending the request, inputting information and checking result. Finally, the platform of human computer interaction is forming.

\subsection{On-line Monitoring Software Design}

This system is only used for the internal environment protection station in Kunming. The function of the system as:

1. The soft is the structure of B/S that the user can login by using browser. 
2. The soft directly shows the position of sewage treatment plants on the map and displays the water quality parameters by using the flexible way of graphic operation.

3. The monitoring center collects monitor data in real-time and uploads the data that had processed and saved into server. According to the requirement of the system, the cycle of collecting data is setting to 10 minute.

4. The way of transmission is GPRS.

5. The statistics statement and chart in real-time can be formed by analysis monitor data.

6. The user login the system by filling in the name and code of users.

7. The system edition is Chinese.

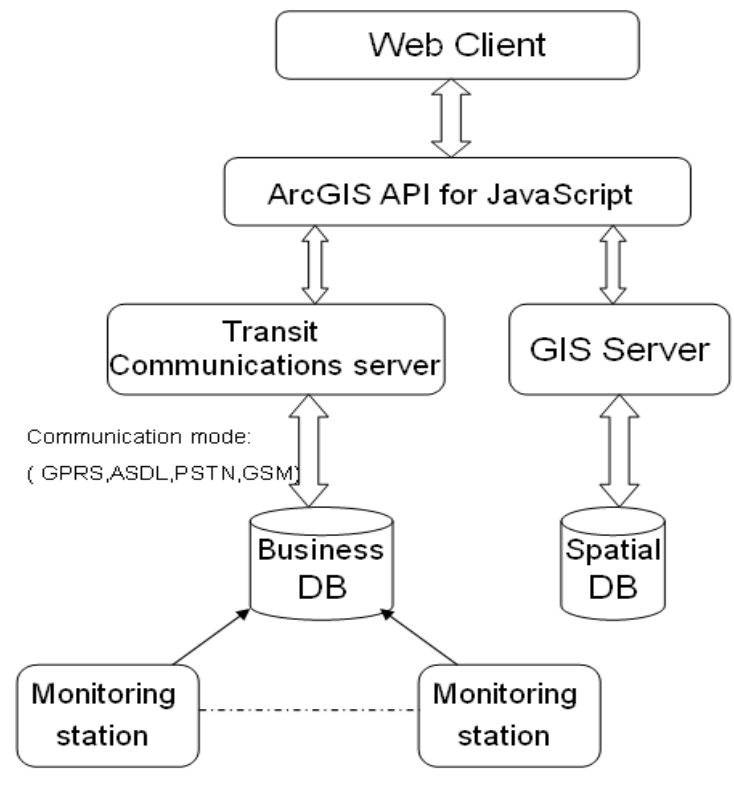

Figure 1. System structure

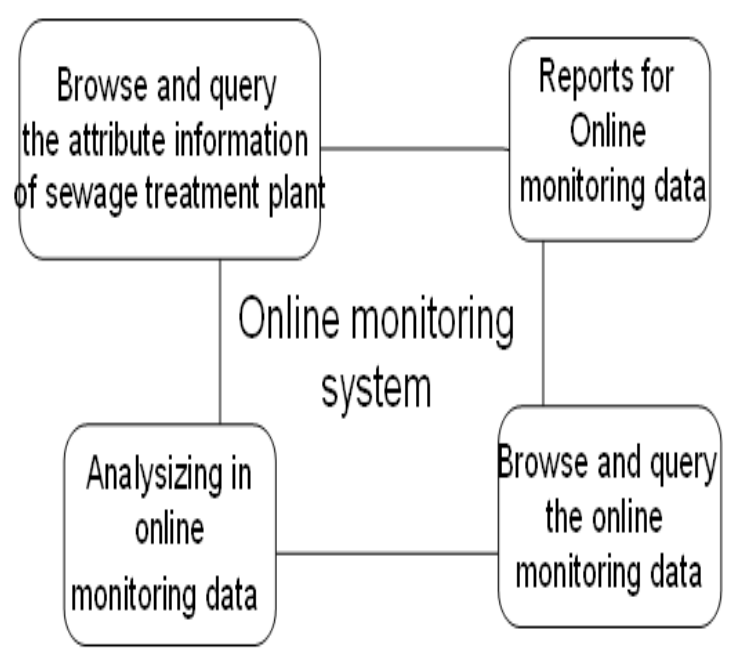

Figure 2. The function of Software system

\section{The introduction of key technology}

\subsection{ArcGIS Server JavaScript API}

ArcGIS Server JavaScript API is GIS application that is provided by ESRI (Environmental Systems Research Institute). GIS application is the feature of the lightweight, high-performance, pure browser. The purpose of ArcGIS Server JavaScript API is build immersive Web GIS by using publishing map service as a tool of location frame and spatial analysis and integrating the others services on the internet. At the same time, this application provides for a lightweight solution to bulid WebGIS application. Through taking advantage of JavaScript API, the client-side applications can call the services provided by ArcGIS Server and realize Map Apps and geoprocessing task.

ArcGIS provides API development mode, which base on the platform for Web of three mainstream client-sides (Adobe Flex, Microsoft Silver light, and JavaScript). The reason for choosing WebService as follows:

1. JavaScript can be supported by all major browsers. It is no need to install any plug-in. However, Flex and Silverlight are needed to install the plug-in.

2. Javascript is of a variety of mature framework (such as Dojo, jQuery, ExtJS, Prototype and so on) makes it possible to improve operating speed of JavaScript.

3. Nowadays, Internet is based on HTTP requests and HTML forms; however, JavaScript is good at controlling the DOM of abstraction layer in HTML.

\subsection{Web Service}

As a common remote method invocation, Web Service achieves interactive between client and server by using the standard and SOAP. This standard is completely based on XML, XSD or the others and independent of the software vendors and platform. WebService mainly focused on the 
communication of heterogeneous system and different language systems. The reasons for choosing WebService as follows:

1. The development platform of client-side and server-side are different. WebService is of inter-platform and cross-web and suitable for setting up open distributed application system in different networks and platforms.

2. It have good interoperability with the other programs. It can realize the communication between different languages because of any language support XML text parsing. For example, Java, C++, Visual Basic or other languages can be communicated with each other.

3. It is of good encapsulation and loose coupling. If client-side knows the feature lists are provided by server-side, any changes can be known by client-side.

\subsection{The working principle of WebService}

WebService is built basing on three roles (Provider, UDDI Registry, and Requester) and three actions (publish, find, and bind). The service of WebService is defined as the provider and publishes it to the UDDI Registry. The requester finds service from UDDI Registry by using find operation and binds with Provider by service. The working principle as shown in figure 3.

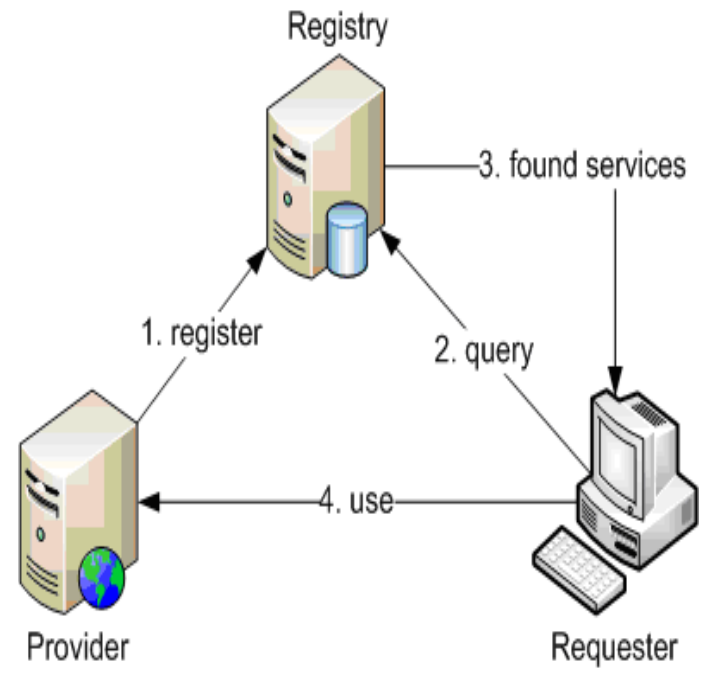

Figure 3. The working principle of WebService

When Requester wants to invoke a service, the service should be found from UDDI Registry. Requester will request to server for calling methods, if the service existing. While the server received the request, it immediately returns an XML document with description of WSDL, which shows all kinds of viable methods. The requester will package these methods into HTTP request with SOAP and send it to server, the Server makes a response and returns SOAP package.

\subsection{The implementation process of WebService client in Java}

When using Web Service in Java, the service should be generate corresponding WSDL file, and put it into HTTP server. According to WSDL file, client stub codes should be generate by using Java tools. The stub packages object request information into SOAP data format and sends request to server-side. The server-side transforms the data when received the request and calls corresponding Java classes.

\section{System implementation}

\subsection{The Browsing and Querying of the constantly water quality monitoring data}

The content of inlet and outlet's chemical index can be shown by clicking any sewage treatment plant on the map. For example, the maximum value, minimum value and the average value of $\mathrm{PH}$, COD, ANDAN and the rate of flow. As shown in figure 4.

\subsection{Analyzing in online monitoring data of sewage treatment}

The changing content of inlet and outlet influences the result of sewage discharge. Therefore, chart analysis is very important. According to these key words (name of sewage treatment, time, specific 
water quality parameters), the charts of inlet and outlet can be generated by the minute, hour, date. At the same time, it can be exported and saved.

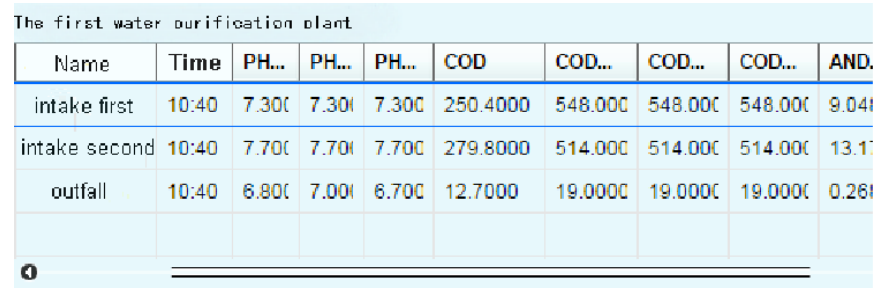

Figure 4. Browsing and querying the monitoring data of water quality in real time

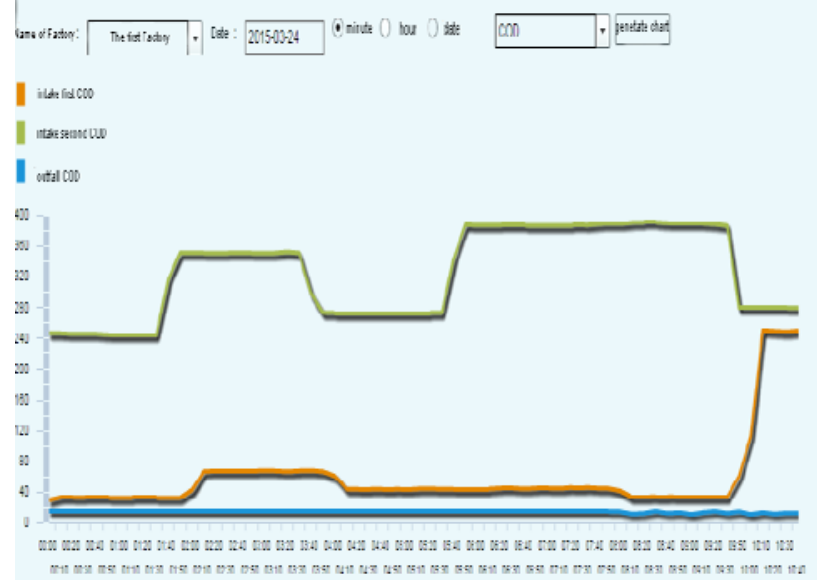

Figure 5. Analysizing in sewage treatment online monitoring data (by minute).

\section{Conclusion}

The system remotely calls transit server data by using Web Service technology and makes statistical analysis by Combining with ArcGIS Server JavaScript API. Finally, the data can be shown by reports or charts. The system changes the traditional mode of scene sampling and analyzing data. The users can be grasp the content and variation of water chemical index timely and accurately and the decision-making information provided for sewage treatment regulators.

\section{References}

[1] Amor V.M.Ines,Ashim Das Gupta, Rainer Loof.Application of GIS and crop growth models inestimating water productivity[J].Agricultural Water Management,2002,54(3):205-225.

[2] Beijing Boda Water Co. Renewable water supply network on-line monitoring system[p].CN 102045381 May 4, 2011.

[3] Electronic Engineering, Beijing University of Posts and Telecommunications. Seeage-line monitoring system design adn application [M].

[4] HE L,Wu J, Jia YM . Research and implementation on JavaScript-based invocating Web Services in browser-side[J].Journal of the Graduate School of the Chinese Academy of Sciences,2007,24(6):801 805

[5] Theodore E.Endreny, Gregory D.Jennings. A Decision Support System for Water Quality Data Augmentation: A Case Study [J].J. of the Am. Water Resources Association, 1999, 71 (4): 363-378.

[6] W3C.Web Services Activity statement[S]. (2006-12-20)[2007-01-30]. 